\title{
OPEN Platelet derived growth factor receptor $\beta$ (PDGFR $\beta$ ) is a host receptor for the human malaria parasite adhesin TRAP
}

\author{
Ryan W. J. Steel ${ }^{1,6,8}$, Vladimir Vigdorovich ${ }^{1,8}$, Nicholas Dambrauskas ${ }^{1}$, Brandon K. Wilder ${ }^{1,7}$, \\ Silvia A. Arredondo ${ }^{1}$, Debashree Goswami ${ }^{1}$, Sudhir Kumar ${ }^{1}$, Sara Carbonetti ${ }^{1}$, \\ Kristian E. Swearingen ${ }^{2}$, Thao Nguyen ${ }^{1}$, Will Betz ${ }^{1}$, Nelly Camargo ${ }^{1}$, Bridget S. Fisher ${ }^{1}$, \\ Jo Soden ${ }^{3}$, Helen Thomas ${ }^{3}$, Jim Freeth ${ }^{3}$, Robert L. Moritz ${ }^{2}$, D. Noah Sather ${ }^{1,4,5 \bowtie} \&$ \\ Stefan H. I. Kappe $\mathrm{K}^{1,4,5 \bowtie}$
}

Following their inoculation by the bite of an infected Anopheles mosquito, the malaria parasite sporozoite forms travel from the bite site in the skin into the bloodstream, which transports them to the liver. The thrombospondin-related anonymous protein (TRAP) is a type 1 transmembrane protein that is released from secretory organelles and relocalized on the sporozoite plasma membrane. TRAP is required for sporozoite motility and host infection, and its extracellular portion contains adhesive domains that are predicted to engage host receptors. Here, we identified the human platelet-derived growth factor receptor $\beta$ (hPDGFR $\beta$ ) as one such protein receptor. Deletion constructs showed that the von Willebrand factor type $A$ and thrombospondin repeat domains of TRAP are both required for optimal binding to hPDGFR $\beta$-expressing cells. We also demonstrate that this interaction is conserved in the human-infective parasite Plasmodium vivax, but not the rodent-infective parasite Plasmodium yoelii. We observed expression of hPDGFR $\beta$ mainly in cells associated with the vasculature suggesting that TRAP:hPDGFR $\beta$ interaction may play a role in the recognition of blood vessels by invading sporozoites.

There were more than 400,000 deaths and 229 million estimated clinical cases of malaria in 2019, the vast majority of which were caused by Plasmodium falciparum and P. vivax ${ }^{1}$. Malaria infection is preceded by $\leq 100$ sporozoites being deposited into the skin with the bite of a female Anopheles mosquito ${ }^{2}$. While in the skin, sporozoites search for blood vessels and invade the vasculature, through which they are transported to the liver. Here, sporozoites traverse liver sinusoidal cells, Kupffer cells and multiple hepatocytes before finally invading one suitable for infection ${ }^{3,4}$. Within the hepatocyte, the parasite transforms and grows as a liver stage, ultimately differentiating into tens of thousands of exoerythrocytic merozoites. Upon egress from the liver, these merozoites infect red blood cells. The ensuing intraerythrocytic infection cycle is responsible for all clinical malaria symptoms, as well as for giving rise to gametocyte stages ready for uptake by a new mosquito vector ${ }^{5-9}$. The sporozoite stage is considered an attractive target for intervention, since preventing parasites from reaching the liver promises to prevent both malarial disease and onwards transmission of the parasite. Thus, efforts are ongoing to identify sporozoite-stage proteins whose function may be disrupted through immune intervention.

Parasite adhesion proteins enabling sporozoite gliding motility, tissue traversal and infection are released from a set of apical organelles, the micronemes and rhoptries ${ }^{10}$. An important sporozoite micronemal protein called the thrombospondin-related anonymous protein (TRAP), is required for both sporozoite motility as well as hepatocyte infection ${ }^{11}$. A small amount of TRAP is released apically from micronemes and subsequently translocated

\footnotetext{
${ }^{1}$ Seattle Children's Research Institute, Seattle, WA, USA. IInstitute for Systems Biology, Seattle, WA, USA. ${ }^{3}$ Retrogenix Ltd, Chinley, High Peak SK23 6FJ, UK. ${ }^{4}$ Department of Pediatrics, University of Washington, Seattle, WA, USA. ${ }^{5}$ Department of Global Health, University of Washington, Seattle, WA, USA. ${ }^{6}$ Present address: Infectious Diseases and Immune Defence Division, The Walter and Eliza Hall Institute of Medical Research, Parkville, VIC 3052, Australia. ${ }^{7}$ Present address: Vaccine and Gene Therapy Institute, Oregon Health and Science University, Beaverton, OR 97006, USA. ${ }^{8}$ These authors contributed equally: Ryan W. J. Steel and Vladimir Vigdorovich ${ }^{\square}$ email: noah.sather@seattlechildrens.org; stefan.kappe@seattlechildrens.org
} 


\begin{tabular}{|c|c|c|}
\hline Protein (database reference) & Domain(s) & Amino acids \\
\hline \multirow[t]{6}{*}{ PfTRAP (PlasmoDB:PF3D7_1335900) } & Full Ectodomain & $26-513$ \\
\hline & VWA + TSR & $26-288$ \\
\hline & VWA & $26-244$ \\
\hline & TSR & $241-292$ \\
\hline & TSR + Repeats & $243-513$ \\
\hline & Repeats & $291-513$ \\
\hline PvTRAP (PlasmoDB:PVX_082735, PlasmoDB:PVP01_1218700) ${ }^{\mathrm{a}}$ & Full Ectodomain & $25-493$ \\
\hline \multirow[t]{2}{*}{ PyTRAP (PlasmoDB:PYYM_1351500) } & Full Ectodomain & $23-752$ \\
\hline & VWA + TSR & $23-281$ \\
\hline PDGF-BB (GenBank: CAG30424.1) & Ectodomain & $82-190$ \\
\hline hPDGFR $\beta$ (GenBank: BC032224.1) & Ectodomain & $33-532$ \\
\hline
\end{tabular}

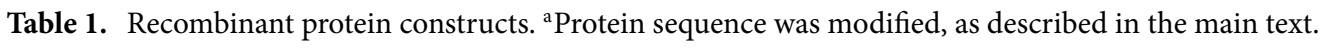

rearward on the sporozoite surface during gliding, thereby acting as an essential link between the sporozoite actin/myosin motor and extracellular substrates ${ }^{12-14}$. In addition to its role in motility, during hepatocyte infection, TRAP becomes largely surface-exposed at the anterior tip of the sporozoite along with other invasion-related proteins, where it is presumably involved in the engagement of host cell receptors ${ }^{15}$. The involvement of TRAP at multiple stages of the sporozoite journey from the inoculation site in the skin to hepatocyte infection in the liver has thus identified it as a promising target for infection-blocking interventions.

The extracellular portion of TRAP contains two adhesive domains that are critical to its function: a von Willebrand factor A (VWA)-like domain with a metal ion-dependent adhesion site (MIDAS), and a thrombospondin repeat domain (TSR domain $)^{16,17}$. Mutations in these domains preserve motility but reduce sporozoite infectivity ${ }^{16}$. The deletion of the VWA domain renders sporozoites non-motile and non-infectious, and this phenotype can be partly reversed by replacement with a distantly related domain ${ }^{17}$. TSR and VWA domains in other organisms are broadly involved in receptor binding, cell-cell recognition and cell adhesion, suggesting a similar role for TRAP in binding host receptors. To date, several proteins have been shown to bind TRAP, yet none of these interactions fully explains the essential role for TRAP during successful infection. Fetuin-A is a hepatocyte-specific receptor shown to partially mediate infection by Plasmodium berghei in vivo ${ }^{18}$. The widely expressed av-containing integrins that function in cell adhesion were recently suggested to mediate sporozoite gliding in the dermis, although this interaction appeared to play no part in hepatocyte infection ${ }^{19}$. Additionally, TRAP has been shown to bind to heparan sulfate proteoglycans (HSPGs) on the surface of hepatocytes ${ }^{20}$. The binding to host receptors has been proposed to follow a 'dual binding system' model in which the VWA and TSR domains of TRAP coordinate host receptor engagement that likely involves conformational change in the receptor upon divalent cation binding to the MIDAS site ${ }^{16,21}$. Indeed, the $P$. falciparum TRAP interaction with av-containing integrins requires divalent cations and a functional MIDAS domain, but is additionally dependent on a 3-amino-acid RGD (Arg-Gly-Asp) integrin-binding motif immediately C-terminal of the TSR domain ${ }^{19}$. Further work has also shown that substitution of the P. berghei TRAP VWA domain with distantly related sequences can partially rescue sporozoite motility and infectivity without a changing tissue tropism ${ }^{17}$. Taken together, these studies suggest that TRAP participates in multiple host interactions that may serve overlapping functions. A more thorough understanding of these interactions is required for better targeted rational vaccine design efforts, given that targeting TRAP with an antibody-based vaccine has achieved only limited success to date ${ }^{22-26}$.

Here we purified the ectodomain of $P$. falciparum TRAP (PfTRAP) and screened for binding to cells overexpressing $>3300$ individual human cell surface receptors. We found that PfTRAP interacts specifically with human platelet-derived growth factor receptor $\beta$ (hPDGFR $\beta$ ) and further investigated this interaction using HEK293F cells overexpressing this receptor. Binding of PfTRAP to hPDGFR $\beta$ was inhibited by monoclonal antibodies directed against PfTRAP or hPDGFR $\beta$. We further studied the domains of PfTRAP that were required for this interaction, and whether the engagement of PDGFR $\beta$ by TRAP is conserved in other Plasmodium species.

\section{Results}

Recombinant $P$. falciparum TRAP interacts with human PDGFR $\beta$. To identify host receptors for PfTRAP, we expressed its full ectodomain (Table 1, Fig. 1A) in HEK293F cells and purified the soluble secreted protein (Suppl. Figure 1B). Using this protein, we undertook a screen of $>4300$ constructs encoding 3345 unique human plasma membrane proteins (Suppl. Table 1) using the Retrogenix Cell Microarray platform. In this screen, individual constructs encoding membrane proteins were reverse-transfected into HEK293 cells resulting in an array of populations overexpressing different targets along with a ZsGreen1-expressing reporter construct. Upon fixation, these arrays were interrogated using fluorescent complexes containing the PfTRAP ectodomain in search of human receptors that demonstrated reproducible binding to PfTRAP. This screen revealed hPDGFR $\beta$ as a PfTRAP-interacting protein (Suppl. Figure 2). We subsequently verified this interaction using a flow-cytometry-based binding assay, in which HEK293F cells overexpressing the hPDGFR $\beta$ fragment (extracellular and transmembrane domains, amino acids 1-561) fused to GFP were stained with fluorescent complexes 
A
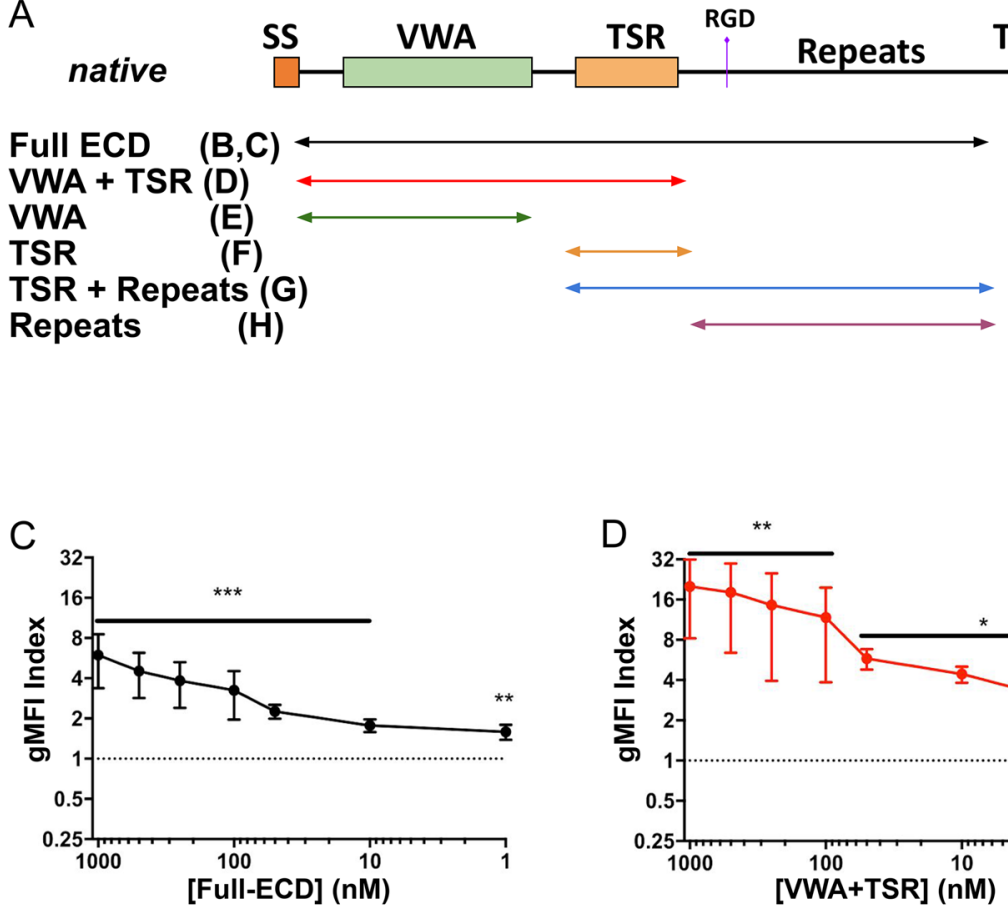

$\mathrm{F}$

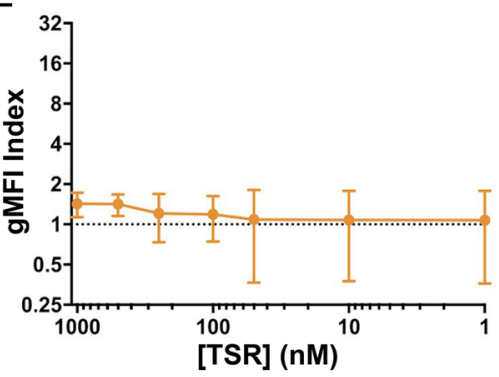

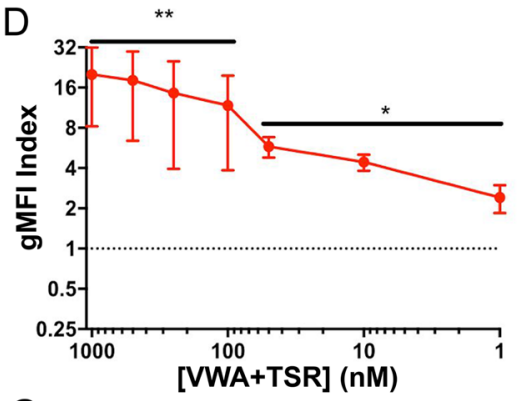

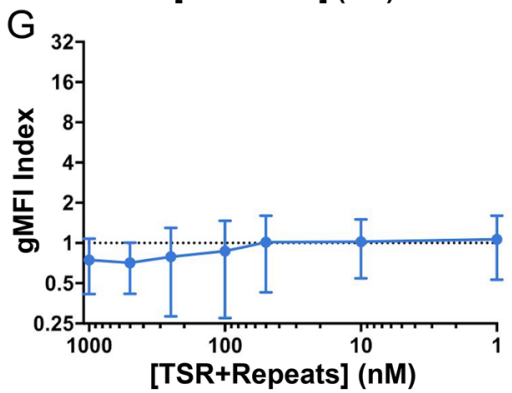

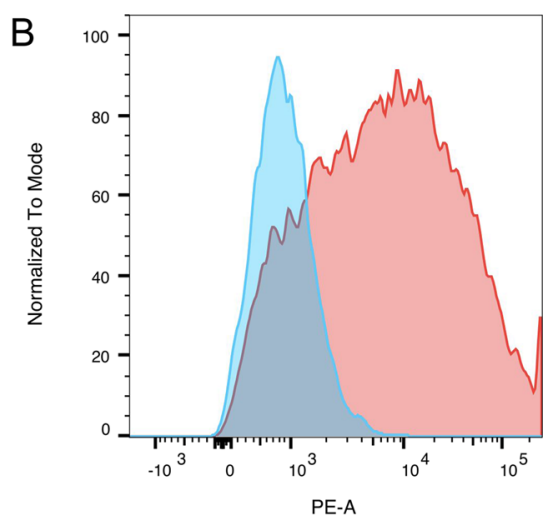

$\mathrm{E}$
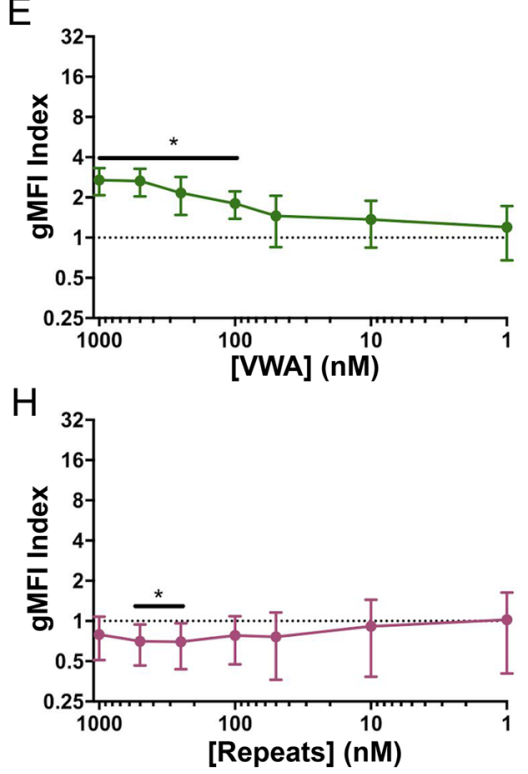

Figure 1. The VWA and TSR domains of PfTRAP act together to bind human PDGFR $\beta$. (A) Overlapping constructs containing various PfTRAP domains are represented as a schematic and were similarly used in further experiments. (B) Biotinylated and fluorescently-labeled PfTRAP ectodomain binds preferentially to the HEK293F cells transfected with hPDGFR $\beta$ (red) over untransfected cells (blue) within the same culture. Titration experiments $(\mathbf{C}-\mathbf{H})$ using increasing dilution of fluorescently labeled fragments of PfTRAP were quantitated as a ratio of binding to transfected versus untransfected cells (gMFI Index) across a 3-log concentration range. Fragments of PfTRAP containing the VWA and TSR domains together bound PDGFR $\beta$ strongly across a 3-log range $(\mathbf{C}, \mathbf{D})$. While the VWA domain alone showed significant binding to hPDGFR $\beta$ at concentrations of $100 \mathrm{nM}$ and above (E), the TSR fragment, the repeat region, or the TSR fragment plus the repeat region did not bind significantly $(\mathbf{F}-\mathbf{H})$. Data are the mean \pm SD from at least five independent experiments. Analysis by one sample t-test compared to the dashed line (gMFI index expected if no specific binding occurs); ${ }^{*} \mathrm{p}<0.05,{ }^{* *} \mathrm{p}<0.01,{ }^{* * *} \mathrm{p}<0.001$. ECD, ectodomain; VWA, von Willebrand factor A domain; TSR, thrombospondin repeat domain.

containing the PfTRAP ectodomain oligomerized around a phycoerythrin-streptavidin core (Fig. 1B). In addition, the screen revealed weak binding of PfTRAP to Crumbs Cell Polarity Complex Component 2 (CRB2) and Ciliary Neurotrophic Factor Receptor (CNTFR), although our subsequent experiments failed to verify these interactions (data not shown).

In order to assess the expression of hPDGFR $\beta$ in tissues most relevant for sporozoite infection, we undertook an immunohistochemical analysis of normal human skin and liver using an antibody specific for hPDGFR $\beta$. In the skin, strong hPDGFR $\beta$ expression was observed in pericytes that undergird the basement membrane of blood vessels (Fig. 2A). To a lesser degree, hPDGFR $\beta$ was also expressed in the basal cell layer of the epidermis. In the liver, more diffuse, low levels of hPDGFR $\beta$ were observed in proximity to major vessels but not in association with liver sinusoids (Fig. 2B).

Optimal engagement of hPDGFR $\beta$ by $P$. falciparum TRAP requires the VWA and TSR domains and is independent from the mechanism of binding to av-containing integrin. We used our flow-cytometry-based binding assay to further investigate the interaction between hPDGFR $\beta$ and PfTRAP (the full gating strategy is shown in Suppl. Figure 3). In addition to the full-length ectodomain, we expressed and 

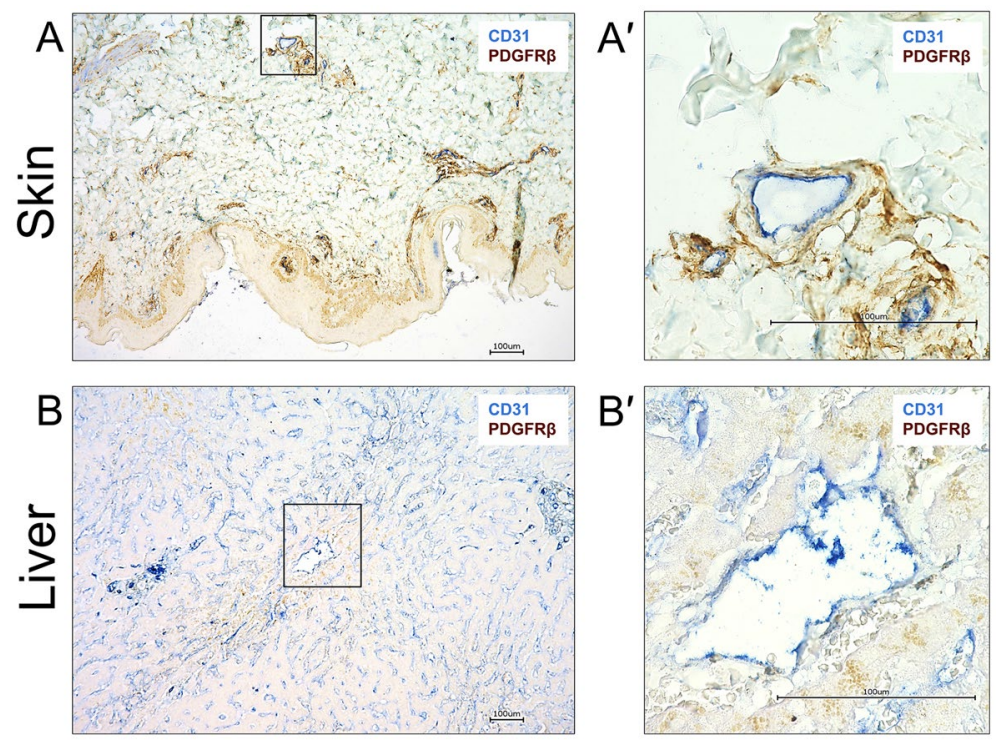

Figure 2. hPDGFR $\beta$ is expressed in the basement membrane of vessels in the skin, and around large blood vessels in the liver. Formalin fixed paraffin embedded sections of normal human skin and liver were stained for CD31 (blue) and PDGFR $\beta$ (brown). (A) Staining for the endothelial marker CD31 could be seen in the lumen of all skin vasculature irrespective of the size. PDGFR $\beta$ was detected surrounding these vascular structures but was not associated with the luminal side of the vessel. Boxed area enlarged in $\mathrm{A}^{\prime}$. (B) CD31 staining was observed in liver sinusoidal endothelial cells (LSEC) all throughout the liver and also in the lumen of major blood vessels. Very low levels of PDGFR $\beta$ staining was detected surrounding large blood vessels but was not associated with the luminal surface of the vessel. No PDGFR $\beta$ staining was seen around LSECs. Boxed area enlarged in B'. Scale bars are $100 \mu \mathrm{m}$.

purified five deletion constructs of PfTRAP (Table 1; Suppl. Figure 1B), as well as the homodimeric form of human platelet derived growth factor B (hPDGF-BB), a known ligand of hPDGFR $\beta$, to be used as a positive control. All purified recombinant proteins were biotinylated and oligomerized using a phycoerythrin-streptavidin conjugate to form a fluorescent staining reagent that was then tested across a 3-log concentration range for binding to HEK293F cells overexpressing hPDGFR $\beta$. Untransfected cells present within each experiment (and trackable due to the lack of GFP signal, which if observed would indicate the expression of the fusion hPDGFR $\beta$ protein), were used to calculate the geometric mean fluorescence intensity (gMFI) ratio with respect to the cells expressing hPDGFR $\beta$-GFP fusion (referred to as gMFI Index).

Full-length PfTRAP ectodomain stained hPDGFR $\beta$-expressing cells in a concentration-dependent manner (Fig. 1C). This binding was enhanced ( $\mathrm{p}<0.01$; Suppl. Figure 4) for the shorter N-terminal VWA + TSR fragment (Fig. 1D). The VWA fragment without the TSR domain bound only modestly (Fig. 1E), while the TSR, alone or in combination with the repeats, or the repeats alone, did not bind to HEK293F cells overexpressing hPDGFR $\beta$ (Fig. 1F,G,H).

We next used monoclonal antibodies (mAbs) directed against PfTRAP and hPDGFR $\beta$ to further evaluate this interaction. Antibodies that bind the PfTRAP VWA domain reduced the binding of both the full-length ectodomain and the VWA + TSR fragment of PfTRAP by approximately $80 \%$ and $90 \%$, respectively, but these mAbs did not affect binding of the known ligand hPDGF-BB to hPDGFR $\beta$ (Fig. 3A). We also employed mAb 2C5, which binds to mouse and human PDGFR $\beta$ (Suppl. Figure 5) and blocks activation of these receptors by their natural ligand PDGF-BB in vitro and in vivo ${ }^{27}$. MAb $2 \mathrm{C} 5$ significantly reduced the binding of full-length ectodomain and the VWA + TSR fragment, and that of the known ligand, hPDGF-BB, to HEK293F cells overexpressing hPDGFR $\beta$ (Fig. 3B). The blocking activity of the antibodies against either partner indicates the specificity of the interaction between hPDGFR $\beta$ and PfTRAP on the cell surface. Despite having shown binding of PfTRAP to cell-associated hPDGFR $\beta$ in our cell-based screen, we were unable to detect binding of PfTRAP to the recombinant soluble form of hPDGFR $\beta$ in vitro (Suppl. Figure 6A), even when measured at high concentration of analyte or in the presence of excess divalent cations (Suppl. Figure 6B).

In a recent report, the ectodomain of $P$. falciparum TRAP was shown to bind av-integrin in a cell-based assay. The mechanism required divalent cations, presumably coordinated by the MIDAS motif of the TRAP VWA domain, and an integrin-binding RGD motif following the TSR domain ${ }^{19}$. We therefore sought to distinguish av-integrin-mediated interactions from those we found for hPDGFR $\beta$. The HEK293F cells used in our assay endogenously express av-integrin (Suppl. Figure 7). We measured PfTRAP binding to untransfected cells under conditions previously shown to inhibit binding to av-integrin. PfTRAP ectodomain, indeed, bound to untransfected cells and this binding was disrupted in the presence of the divalent cation chelator ethylenediaminetetraacetic acid (EDTA) as well as by anti- $\alpha$-integrin antibodies (Fig. 4A,B), in agreement with previous findings ${ }^{19}$. Furthermore, our PfTRAP VWA + TSR fragment lacks the RGD motif that is important for av-integrin binding found 20 amino acids following the TSR domain, and consequently did not bind significantly to untransfected 
A

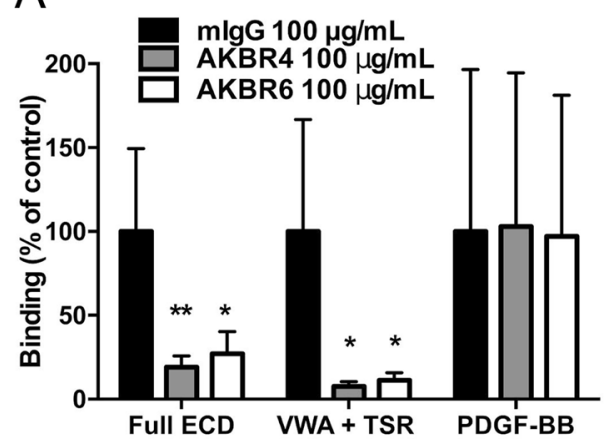

B

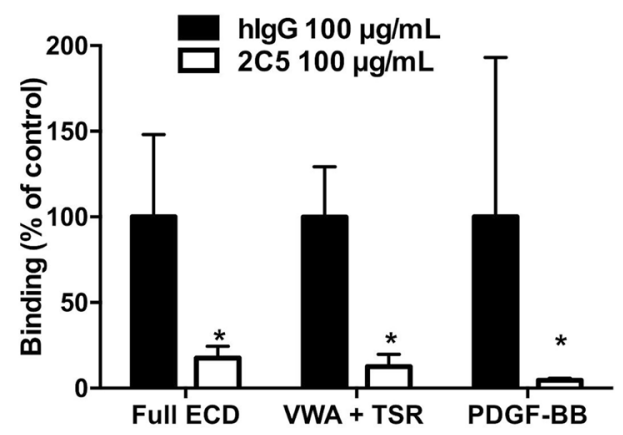

Figure 3. PfTRAP interaction with human PDGFR $\beta$ can be disrupted by parasite and host directed antibodies. (A) Anti-PfTRAP mAb AKBR4 or AKBR6 was incubated with $1000 \mathrm{nM}$ PfTRAP full ectodomain (ECD) or VWA + TSR fragment, or $10 \mathrm{nM}$ of the known PDGFR $\beta$ ligand hPDGF-BB for $30 \mathrm{~min}$ before addition to hPDGFR $\beta$ transfected cells in the flow assay. Binding of the Full ECD and VWA + TSR fragments was reduced by approximately $80 \%$ and $90 \%$, respectively, while binding of human PDGF-BB was unaffected. (B) hPDGFR $\beta$ transfected cells were incubated with anti-PDGFR $\beta$ mAb 2 C 5 for 30 min before addition of staining reagents as above with analysis by flow cytometry. Blocking hPDGFR $\beta$ inhibited binding of both PfTRAP fragments as well as the known ligand human PDGF-BB. Data are normalized to appropriate isotype controls and represent the mean \pm SD from four independent experiments. Analysis by One-way ANOVA with Dunnet's multiple comparisons test (A), and Mann Whitney test (B); ${ }^{\star} \mathrm{p}<0.05,{ }^{* *} \mathrm{p}<0.01$ compared to the isotype control.

A

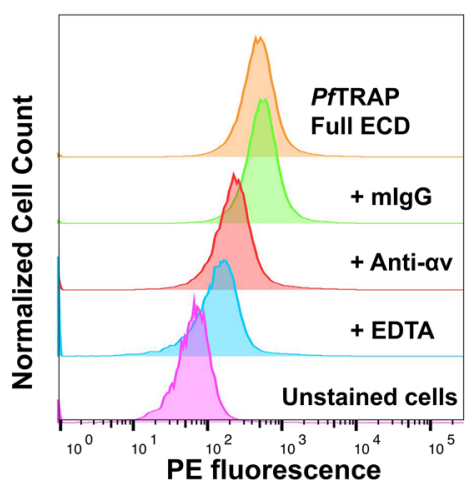

C

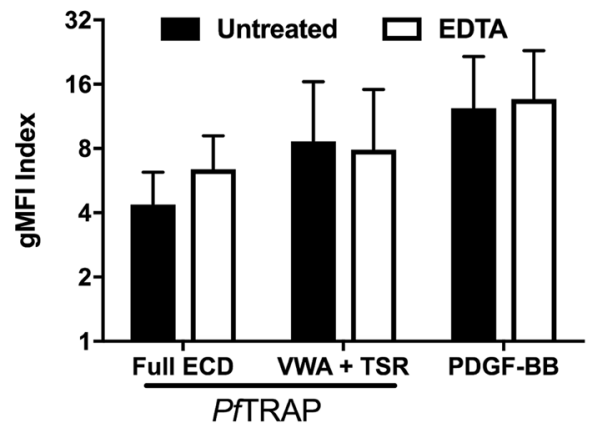

B
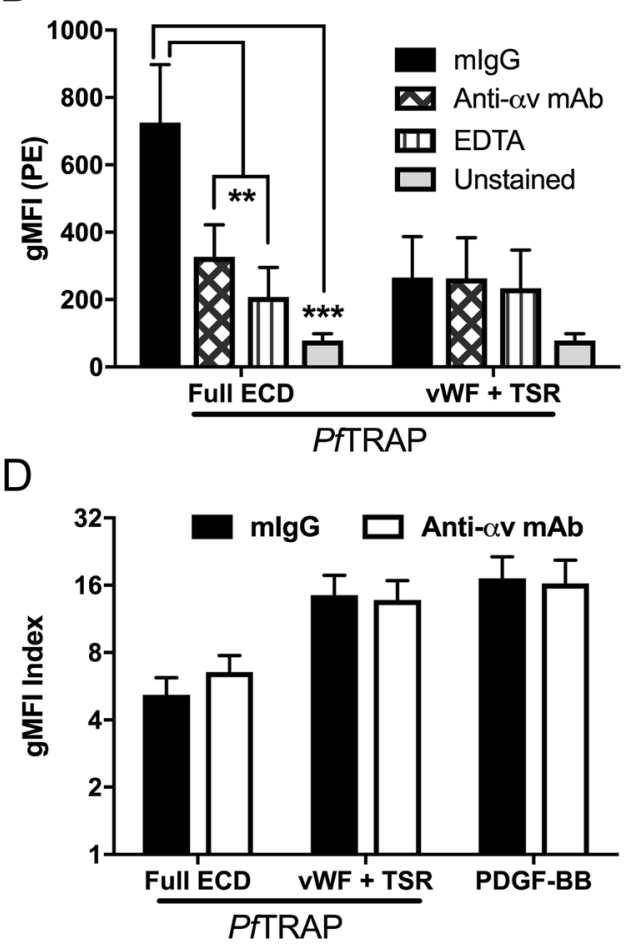

Figure 4. Divalent cations and $\alpha v$-integrin do not contribute to PfTRAP binding of human PDGFR $\beta$. (A, B) PfTRAP ectodomain (Full ECD) staining reagent at $1000 \mathrm{nM}$ bound to untransfected HEK293F cells. This binding was inhibited by both $10 \mathrm{mM}$ EDTA and $10 \mu \mathrm{g} / \mathrm{mL}$ of anti-av-integrin $\mathrm{mAb}$, but not an isotype control antibody. (B) The VWA + TSR fragment of PfTRAP did not bind significantly to untransfected cells and was not affected by EDTA or anti-av-integrin antibodies. (C, D) Presence of $10 \mathrm{mM}$ EDTA or $10 \mu \mathrm{g} / \mathrm{mL}$ anti-av-integrin $\mathrm{mAb}$ did not significantly affect the binding of PfTRAP ECD $(1000 \mathrm{nM})$, the VWA + TSR fragment $(1000 \mathrm{nM})$ or the natural ligand PDGF-BB $(10 \mathrm{nM})$ to HEK293F cells transfected with human PDGFR $\beta$. Data are the mean \pm SD from at least three independent experiments. Analysis by One-way ANOVA with Dunnet's multiple comparisons test (B), and paired t-test $(\mathbf{C}-\mathbf{D}) ;{ }^{*} \mathrm{p}<0.05,{ }^{* *} \mathrm{p}<0.01,{ }^{* * *} \mathrm{p}<0.001$. 

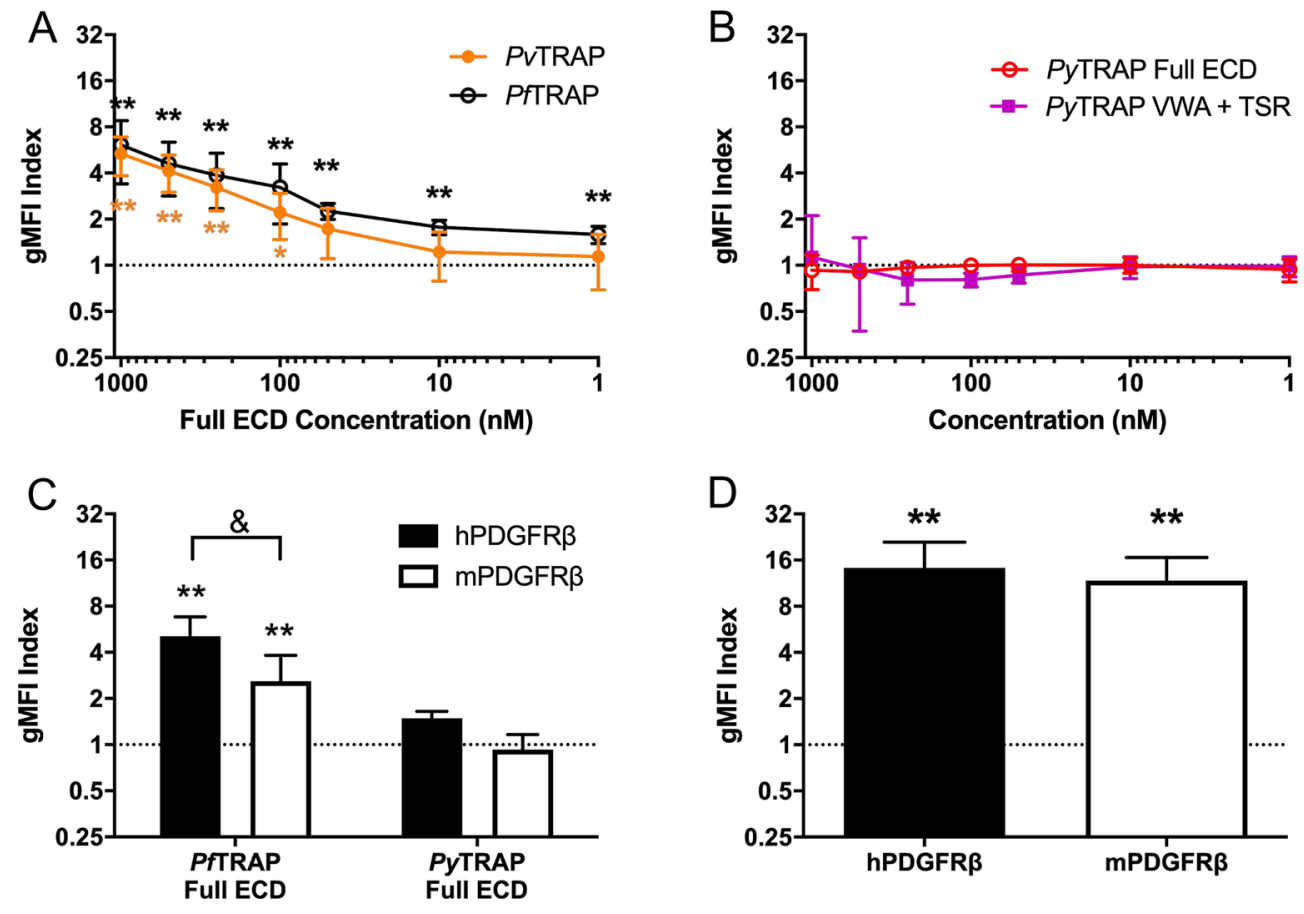

Figure 5. Engagement of PDGFR $\beta$ by TRAP distinguishes human and rodent infective Plasmodium species and is restricted at the level of TRAP. (A) PvTRAP ectodomain (ECD) staining reagent bound HEK293F cells transfected with human PDGFR $\beta$ (hPDGFR $\beta$ ) in a concentration dependent manner that was not different from the binding of PfTRAP ECD. (B) PyTRAP ECD or a fragment containing the VWA + TSR domains did not bind to HEK293F cells transfected with mouse PDGFR $\beta$ (mPDGFR $\beta$ ) at any concentration tested. (C) PfTRAP and PyTRAP ECD were tested at $1000 \mathrm{nM}$ for binding to both hPDGFR $\beta$ and mPDGFR $\beta$ transfected HEK293F cells. PfTRAP ECD bound to both receptors, although less strongly to mPDGFR $\beta$, while PyTRAP ECD did not specifically bind to either receptor. (D) Known ligand for hPDGFR $\beta$ and mPDGFR $\beta$, human PDGF-BB bound to both receptors at $10 \mathrm{nM}$. Data are the mean \pm SD from at least three independent experiments. Asterisks indicate analysis by one sample t-test compared to the dashed line (gMFI index expected if no specific binding occurs), ${ }^{*} \mathrm{p}<0.05,{ }^{* *} \mathrm{p}<0.01$; ampersand indicates analysis by t-test, ${ }^{\mathrm{a}} \mathrm{p}<0.05$.

cells (Fig. 4B). This indicated that the binding to untransfected cells potentially was due to the interaction with the av-integrin. We next tested the effect of EDTA and anti-av-integrin antibodies on PfTRAP binding to cells transfected with hPDGFR $\beta$. Both the full ectodomain and the VWA + TSR constructs of PfTRAP showed strong binding that was not affected by EDTA or anti-av-integrin antibodies (Fig. 4C,D), indicating that the binding measured in hPDGFR $\beta$-transfected cells was not due to binding endogenous av-integrin, but was specific to hPDGFR $\beta$.

Collectively these data show that optimal binding of PfTRAP to hPDGFR $\beta$ is mediated by the VWA and TSR domains acting together. We also show that this interaction can be disrupted using host- and parasite-directed antibodies and demonstrate that the binding of PfTRAP to hPDGFR $\beta$-expressing cells is distinguishable from the recently discovered interaction with av-integrins ${ }^{19}$.

We next set out to test whether the TRAP:PDGFR $\beta$ interaction is important for sporozoite infection of the mammalian host. To this end, we tested passive transfer of blocking mAb 2C5 (which also blocks mouse mPDGFR $\beta$ function in vivo) into mice, followed by sporozoite challenge via mosquito bite using the rodent malaria parasite $P$. yoelii (Suppl. Figures 5C,D). Interestingly, mPDGFR $\beta$ blockade with mAb 2 C5 did not have a significant effect on sporozoite infection of mice when compared to control either in terms of decreased liver burden (Suppl. Figure 5C) or delay to blood-stage patency (Suppl. Figure 5D). This result precipitated a further investigation into whether the TRAP:PDGFR $\beta$ interaction is conserved among Plasmodium species.

Engagement of PDGFR $\beta$ by TRAP is conserved across human-infective Plasmodium species, but not in a rodent-infective Plasmodium species. We produced a recombinant ectodomain fragment for the human-infective malaria parasite P. vivax (PvTRAP), as well as full ECD and N-terminal adhesive domains (VWA + TSR) fragments for the rodent infective malaria parasite P. yoelii (PyTRAP) (Table 1; Suppl. Figure 1C,D) and tested them for binding to cells overexpressing PDGFR $\beta$ of mouse (mPDGFR $\beta$ ) and human (hPDGFR $\beta$ ) origin. Both PyTRAP and PvTRAP sequences are significantly divergent from that of PfTRAP, with the ectodomains sharing $23 \%$ and $40 \%$ amino acid sequence identity with PfTRAP, respectively. Binding of PvTRAP to hPDGFR $\beta$ was seen in a concentration-dependent manner that was not significantly different from the binding of PfTRAP (Fig. 5A). We, however, observed no binding of either PyTRAP ectodomain or 
VWA + TSR to mPDGFR $\beta$ (Fig. 5B). This might explain why we observed that blocking the interaction in a rodent malaria infection model had no discernable effect on sporozoite infection (Suppl. Figure 5C,D).

Finally, we assessed the cross-interaction of PyTRAP with hPDGFR $\beta$, and PfTRAP with mPDGFR $\beta$. The PfTRAP fragment bound significantly to MPDGFR $\beta$, albeit only at approximately $50 \%$ of the level of binding to hPDGFR $\beta$ (Fig. 5C). No specific binding of PyTRAP to either mPDGFR $\beta$ or hPDGFR $\beta$ was observed. Both human and mouse PDGFR $\beta$ bound the human PDGF-BB ligand to a similar extent (Fig. 5D), ruling out gross discrepancies in protein localization or expression levels affecting these results.

\section{Discussion}

Over the last decade, numerous Plasmodium proteins have been identified that play a role in the sporozoites' journey from the site of deposition in the skin to their destination in the liver and their ultimate infection of host hepatocytes $^{28}$. Furthermore, host hepatocyte surface proteins $\mathrm{CD} 81^{29}, \mathrm{SR}-\mathrm{B} 1^{30}$ and EphA2 $2^{31}$ have been described as critical factors for infection of hepatocytes, although this has been recently challenged for EphA $2^{32}$. Yet, linking host receptors to sporozoite ligands has been exceedingly difficult, and sporozoite protein/host cell protein interaction pairs that might play a role in host infection remain virtually unknown. We have herein identified PDGFR $\beta$ as a novel host receptor for the essential sporozoite adhesin TRAP by screening $>3300$ human plasma membrane proteins in the most comprehensive binding screen for this important adhesin to date. The PDGFR $\beta$ :TRAP interaction can be blocked with mAbs to either the parasite protein or the host receptor, requires both TRAP VWA and TSR domains for efficient binding, and is independent of the TRAP MIDAS and RGD motifs required for its binding to $\alpha v \beta 3$-integrin ${ }^{19}$. Intriguingly, the PDGFR $\beta$ interaction with TRAP is conserved among the major human-infective $P$. falciparum and $P$. vivax parasites but not for the rodent-infective parasite $P$. yoelii.

PDGFR $\beta$ plays a major role in angiogenesis and maintenance of vascular integrity, and was shown to be expressed by lymphatic endothelial cells as well as pericytes supporting endothelial cells during development ${ }^{33-35}$. Additionally, PDGF receptors have long been recognized to play an important role in cutaneous wound healing ${ }^{36}$. Consistent with these published vascular expression patterns, we detected PDGFR $\beta$ expression in a perivascular distribution in human skin. Thus, it is possible that the TRAP:PDGFR $\beta$ interaction may have a role in mediating sporozoite recognition of the vasculature. However, our findings indicate that further functional studies for TRAP:PDGFR $\beta$ interactions must be undertaken in model systems that recapitulate the human vascular compartment.

Binding of recombinant PfTRAP to hPDGFR $\beta$ was detectable in our cell-based assay in a concentrationdependent manner. However, we were unable to detect binding of recombinant TRAP to the purified soluble form of PDFGR $\beta$, despite detecting a robust interaction with its known ligand PDGF-BB. It is possible that the conformation of the membrane-anchored PDGFR $\beta$ differs from that of its soluble counterpart, or that the TRAP interaction requires the presence of additional, unidentified proteins associated with PDGFR $\beta$.

Our findings complement the recent study by Dundas and colleagues ${ }^{19}$ in which $a v \beta 3$ integrin was identified as a host receptor for TRAP. It is likely that this interaction was not detected in our human surface protein screen because only individual proteins (i.e., either $\alpha v$ chain or $\beta 3$ chain alone) were overexpressed. We have independently validated the binding of PfTRAP to av-containing integrins, which have basal expression in our cell-based system. However, we have shown that TRAP binding to hPDGFR $\beta$ was distinct as indicated by our findings that (i) binding to hPDGFR $\beta$ did not require the RGD motif containing repeat region, (ii) binding was insensitive to anti- $\alpha \mathrm{v}$ antibodies and (iii) unlike the interaction with av-containing integrins ${ }^{19}$, binding did not require divalent cations. Furthermore, the interaction with hPDGFR $\beta$ was conserved for TRAP of $P$. falciparum and $P$. vivax. In contrast, the interaction between integrin $\alpha v \beta 3$ and the TRAP of $P$. vivax had not been observed by Dundas et al. ${ }^{19}$. Thus, it is highly likely that the interactions of TRAP with $\alpha v \beta 3$ and PDGFR $\beta$ are distinct and might serve different biological functions during the infection process. The notion that TRAP may interact with multiple binding partners is further supported by recent work by Klug et al., who found that substitution of the TRAP VWA domain with distantly related sequences of this domain from other species can rescue noninfectious VWA-deletion mutants ${ }^{17}$.

In order to directly establish the potential biological importance of the TRAP:PDGFR $\beta$ interaction, we initially attempted to block the interaction in vivo and measure the effect on sporozoite transmission success, using the $P$. yoelii mouse malaria model. Interestingly, however, $P$. yoelii TRAP failed to show significant binding to hPDGFR $\beta$ or mPDGFR $\beta$ (Fig. 5C). Indeed, PyTRAP shares only $23 \%$ amino acid sequence identity with PfTRAP, which might explain the lack of binding. Yet, sequence divergence alone might not fully account for lack of binding, as binding to PDGFR $\beta$ with PvTRAP is functionally conserved despite only $40 \%$ shared amino acid sequence identity with PfTRAP. Thus, it is probable that the interaction between TRAP and PDGFR $\beta$ is not conserved in $P$. yoelii, and this will not be an adequate model to study the biology of TRAP:PDGFR $\beta$ interaction. Thus, our findings are also adding to the emerging picture of differences in the conservation of host receptor engagement by orthologous effector proteins between human-infective and rodent-infective Plasmodium species ${ }^{19,37}$.

Combined with recent studies, our findings provide a more complete understanding of the potential molecular interactions of TRAP with host receptors. While the biological consequences of the TRAP:PDGFR $\beta$ interaction remain to be fully deciphered, our work provides a novel molecular interaction lead that could mediate the initial infection of the human host by malaria parasites.

\section{Methods}

Recombinant protein production. Production of recombinant proteins in suspension HEK293F cells cultures was performed, as previously described ${ }^{31,38,39}$. Briefly, each expression construct contained codonoptimized sequences encoding a tissue plasminogen activator signal peptide ${ }^{40}$, the full extracellular domain or subdomains from P. yoelii TRAP (PyTRAP) (PlasmoDB:PYYM_1351500), P. falciparum TRAP (PfTRAP) 


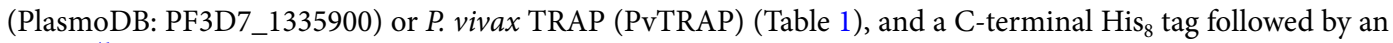
AviTag ${ }^{41}$ cloned into the pcDNA-3.4 vector backbone (Thermo Fisher, Waltham, MA, USA). Constructs encoding tagged human PDGF-B (residues 82-190) (purified as homodimeric hPGDF-BB), and the tagged ectodomain of hPDGFR $\beta$ (residues 33-532) were similarly created.

The PvTRAP sequence was based on that observed by proteomic analysis of a Thai $P$. vivax salivary gland sporozoite sample bearing the VK247 haplotype of CSP ${ }^{42}$. Protein sequence polymorphisms in the field isolate were identified by augmenting the $P$. vivax Sal-1 genome ${ }^{43}$ used for the protein sequence database search with the $P$. vivax $\mathrm{P} 01$ genome ${ }^{44}$ as well as sequence polymorphisms observed in RNA-seq analyses of 19 different Thai field isolates ${ }^{45}$. Additionally, $\mathrm{S} \rightarrow \mathrm{A}$ and $\mathrm{T} \rightarrow \mathrm{A}$ substitutions were made in NX[S/T] motifs to prevent N-linked glycosylation, as this modification has not been shown to occur in Plasmodium ${ }^{46,47}$ and is absent from TRAP in vivo ${ }^{42}$.

Constructs were introduced into HEK293F cells by high-density transfection with PEI MAX 40 K (Polysciences, Inc., Warrington, PA, USA $)^{48,49}$, and culture supernatants containing the recombinant proteins were harvested after 5 days. Proteins were purified by $\mathrm{Ni}^{2+}$-affinity chromatography followed by size-exclusion chromatography. Subsequently, a BirA biotin-protein ligase kit (Avidity, LLC; Aurora, CO, USA) was used to biotinylate protein samples, as previously described ${ }^{39}$. Biotinylated proteins were re-purified by size-exclusion chromatography to remove free biotin, and multimerized using streptavidin-phycoerythrin (ProZyme) by mixing 1 molarequivalent of streptavidin-phycoerythrin with 4 molar-equivalents of biotinylated protein.

Retrogenix cell microarray screen. Screening for PfTRAP binding proteins was performed using the Retrogenix Cell Microarray technology. Initially 4336 expression vectors, each encoding a unique human plasma membrane protein and the ZsGreen1 reporter constructs, were pre-spotted on glass slides using a QArray mini microarrayer (Genetix, UK). Human HEK293 cells were grown over the arrays and reverse-transfected, resulting in the over-expression of each membrane protein and ZsGreen1. Slides were then incubated with QDot ITK Carboxyl Quantum Dots, 585 nM (Life Technologies cat \# Q21311MP) which were pre-coupled with Ni-NTA and coated with His-tagged PfTRAP, giving a final PfTRAP concentration of $100 \mathrm{nM}$. PfTRAP binding proteins were identified by fluorescence imaging (at $~ 500 \mathrm{~nm}$ wavelength for ZsGreen1 and at $\sim 585 \mathrm{~nm}$ for QDots) using an Ettan DIGE imager (GE Healthcare) and analysed using ImageQuant software (GE). For confirmation and specificity testing, expression vectors encoding the PfTRAP binding proteins were re-spotted on new slides, and HEK293 cells were reverse-transfected. Slides were then treated with the same Ni-NTA-coupled QDots coated with His-tagged PfTRAP as before, or with His-tagged PDL1 (each at $100 \mathrm{nM}$ final concentration), or with no ligand conjugated. Interactions were analysed by fluorescence imaging using an Ettan DIGE Imager.

Protein binding measurements. Protein interactions were analyzed using biolayer interferometry on an Octet $\mathrm{QK}^{\mathrm{e}}$ instrument (ForteBio). Streptavidin (SA) biosensors were used to immobilize biotinylated recombinant ligand molecules followed by equilibration in $10 \times$ Kinetics Buffer (PBS $+0.1 \%$ BSA, $0.02 \%$ Tween-20 and $0.05 \%$ sodium azide), detection of association of recombinant analyte proteins diluted in the same buffer, and detection of dissociation in analyte-free $10 \times$ Kinetics Buffer.

Experiments requiring the use of salts of divalent metals required phosphate to be substituted with HEPES (since phosphate formed poorly soluble salts with several of the metal ions tested). In those cases, we used HBS-P (20 mM HEPES, pH 7, $150 \mathrm{mM} \mathrm{NaCl}, 0.1 \%$ BSA, 0.02\% Tween-20) as the base buffer.

Flow cytometry binding assays. To further analyze TRAP binding, biotinylated recombinant TRAP proteins (Table 1) were oligomerized around a streptavidin-conjugated Phycoerythrin (PE) (PJRS2, Prozyme) creating TRAP-PE staining complexes. Sequence verified human (clone IMAGE:5309813) and mouse (clone IMAGE:30060666) cDNAs for PDGFR $\beta$ were obtained from the Dharmacon Mammalian Gene Collection (GE Healthcare) for creation of surface-expressed GFP fusion receptor constructs. The 5 ' open reading frame fragment, including the transmembrane region and a short intracellular linker (encoding amino acids 1-561 of either human or mouse cDNA), was cloned along with a GFP-encoding fragment into a pcDNA3.4 expression vector (Thermo Fisher), resulting in the intracellular tyrosine kinase domain of the receptor being replaced with Cycle3 GFP. Approximately $3 \times 10^{6}$ HEK293F cells were transfected with one of these constructs using 293fectin Transfection Reagent (Thermo Fisher) or 293-Free (EMD Millipore), according to the manufacturer's instructions and maintained in FreeStyle 293 serum-free medium (Thermo Fisher). Two days after transfection approximately $5 \times 10^{5}$ cells were transferred to 96 -well plates, pelleted at $500 \times \mathrm{g}$ for $3 \mathrm{~min}$, then resuspended and incubated with various concentrations of protein complexes in FACS buffer (PBS + 2\% FBS) for 30 min on ice. Cells were then washed three times with FACS buffer before analysis without fixation on an LSR II Flow Cytometer (Becton Dickinson) using FlowJo (TreeStar). Specificity for binding to the transfected receptor was measured by the geometric mean fluorescent intensity (gMFI) of PE on transfected $\left(\mathrm{GFP}^{+}\right)$cells normalized to the untransfected $\left(\mathrm{GFP}^{-}\right)$cells in the same well, termed the gMFI Index. Experiments with the known ligand for PDGFR $\beta$, a dimer of the human Platelet-Derived Growth Factor-B (hPDGF-BB), and mock transfected cells were included as controls in the assays. Experiments testing the role of divalent metals in binding were done in presence of $10 \mathrm{mM}$ EDTA. Blocking of the integrin av binding was performed using the mouse monoclonal 272-17E6 antibody (Abcam).

Recombinant antibody production. For PfTRAP blocking experiments monoclonal antibodies recognizing PfTRAP were produced as previously described ${ }^{39}$. Briefly, BALB/cJ mice were immunized intramuscularly three times, three weeks apart, with $20 \mu \mathrm{g}$ of PfTRAP full ectodomain formulated in Adjuplex (Sigma Aldrich). Two weeks following the final immunization spleens from PfTRAP immunized mice were harvested, dissociated, and antigen-specific B cells sorted by FACS into 96-well plates for subsequent culture. Sorted cells 
were stimulated to secrete antibody and supernatants screened for antigen-binding antibodies by ELISA. Antibody sequences encoding the variable regions of heavy and light chains were rescued from the positive wells using RT-PCR and fused to constant-region encoding DNA fragments to build expression constructs.

For PDGFR $\beta$ receptor blocking experiments, a previously published monoclonal antibody identified from a phage display library (clone 2C5) and capable of neutralizing both mouse and human PDGFR $\beta^{27}$ was expressed as a recombinant hIgG1. Briefly, the variable heavy- and light-chain sequences were obtained from Creative Biolabs (NY, USA) and cloned directly into antibody expression vectors.

Antibodies were expressed using the HEK293F culture, as described above for protein production. Following 5 days in culture, supernatants were cleared by centrifugation, supplemented with $\mathrm{NaCl}(+350 \mathrm{mM}$, final concentration), and applied over Protein G Sepharose Fast Flow (GE Lifesciences). Protein was eluted using $0.1 \mathrm{M}$ glycine ( $\mathrm{pH}$ 2.7), and the $\mathrm{pH}$ was immediately neutralized using $1 \mathrm{M}$ dibasic sodium phosphate.

Immunohistochemistry. Localization of PDGFR $\beta$ in formalin-fixed paraffin-embedded normal human skin and liver sections (Amsbio, MA, USA) was performed using a EnVision G2 Doublestain System, Rabbit/ Mouse (Agilent). This staining procedure can be used for sequential double staining of two different antigens with primary antibodies raised in the same species by incorporating a blocking step between the first and second antibody incubations. Briefly, sections were deparaffinized and rehydrated by standard methods, antigens retrieved by immersion in boiling $10 \mathrm{mM}$ sodium citrate ( $\mathrm{pH}$ 6.0) for $10 \mathrm{~min}$, and endogenous peroxidase activity quenched according to the manufacturer's instructions. Sections were then blocked and permeabilized in Protein Block Serum-Free (Agilent) for $20 \mathrm{~min}$ at room temperature before incubation with primary rabbit monoclonal antibody against PDGFR $\beta$ (Abcam; clone Y92). Sections were washed twice in TBS-Tween $0.05 \%$ (20 mM Tris $\mathrm{pH} \mathrm{8,} 150 \mathrm{mM} \mathrm{NaCl}, 0.05 \%$ Tween-20) for $5 \mathrm{~min}$. Polymer/HRP (Agilent) was added to the sections and sections were incubated for $30 \mathrm{~min}$ at room temperature in the dark. Sections were washed twice with TBS-Tween $0.05 \%$ for 5 min each. DAB chromogen (diluted 1 drop every $1 \mathrm{~mL}$ DAB buffer) was added to the sections, and slides were incubated in the dark for $20 \mathrm{~min}$. Sections were washed with distilled water to stop the reaction. For staining of vasculature, sections were stained with the rabbit monoclonal antibody against the endothelial marker CD31 (Abcam; clone EPR3094). Sections were washed with TBS-Tween 0.05\% for 5 min and rabbit/mouse link (Agilent) was added to slides and incubated for $15 \mathrm{~min}$ at room temperature in the dark. Sections were washed twice in TBS-Tween $0.05 \%$ for $5 \mathrm{~min}$ and polymer-AP was added. Sections were incubated for $30 \mathrm{~min}$ at room temperature in the dark followed by two washes with TBS-Tween $0.05 \% 5 \mathrm{~min}$. To visualize CD31, Vector Blue substrate (Vector Laboratories) was prepared according to manufacturer's instructions and added to the sections for $20 \mathrm{~min}$. Reaction was stopped with water. PDGFR $\beta$ straining (brown) and CD31 staining (blue) were visualized and imaged using the Keyence BZ-X710 microscope.

Mosquito rearing and sporozoite production. Anopheles stephensi mosquitoes were reared according to standard protocols and adult females were infected with Plasmodium parasites 3-7 days after their emergence. For P. yoelii sporozoite production, female 6-8-week-old Swiss Webster mice were injected with blood stage 17XNL parasites constitutively expressing GFP/Luciferase $(P y \mathrm{GLuc})^{50}$ to begin the growth cycle, and infected mice were used to feed adult female Anopheles stephensi mosquitoes after gametocyte exflagellation was observed. Blood-fed mosquitoes were then maintained according to standard protocols before their use in animal challenge studies.

mAb 2C5 passive transfer protection studies. All experiments involving animals were performed in strict adherence to the appropriate guidelines and regulations, and under protocols approved by the Institutional Animal Care and Use Committee at the Seattle Children's Research Institute. Mice received two, 800- $\mu \mathrm{g}$ injections of $\mathrm{mAb} 2 \mathrm{C} 5$ or isotype control hIgG before infectious mosquito bite challenge. The first was i.p. $18 \mathrm{~h}$ before challenge to allow distribution of the antibody throughout the tissues, while the second was i.v. $1 \mathrm{~h}$ before to ensure a high serum antibody concentration at the time of challenge. Host directed antibodies against CD81 have been shown to prevent malaria infection with a single dose of $800 \mu \mathrm{g}$ the day before infection ${ }^{51}$. Mouse infection and analysis of malaria liver stage burden was as previously described ${ }^{50,52}$. Briefly, BALB/cJ mice were anesthetized and infected with the bites of 20 PyGLuc infected mosquitoes, respectively. Malaria liver stage burden was measured at the end of liver stage development ( $45 \mathrm{~h}$ post infection) using an In Vivo Imaging System (IVIS) to determine if blocking PDGFR $\beta$ resulted in lower malaria liver stage infection. From day 3 post infection, mice were monitored daily for the presence of erythrocytic stages by Giemsa staining of thin blood smears. The study was carried out and is presented here in compliance with the ARRIVE guidelines.

Statistical analysis. All statistical analyses were performed using GraphPad Prism software (version 6), and differences considered statistically significant when $\mathrm{p}$ was $<0.05$.

Received: 25 February 2021; Accepted: 13 May 2021

Published online: 31 May 2021

\section{References}

1. Geneva: World Health Organization. World malaria report 2020: 20 years of global progress and challenges (2020).

2. Medica, D. L. \& Sinnis, P. Quantitative dynamics of Plasmodium yoelii sporozoite transmission by infected anopheline mosquitoes. Infect. Immun. 73, 4363-4369 (2005). 
3. Amino, R. et al. Quantitative imaging of Plasmodium transmission from mosquito to mammal. Nat. Med. 12, 220-224 (2006).

4. Ejigiri, I. \& Sinnis, P. Plasmodium sporozoite-host interactions from the dermis to the hepatocyte. Curr. Opin. Microbiol. 12, 401-407 (2009).

5. Frevert, U. et al. Intravital observation of Plasmodium berghei sporozoite infection of the liver. PLoS Biol. 3, e192 (2005).

6. Mota, M. M. et al. Migration of Plasmodium sporozoites through cells before infection. Science 291, 141-144 (2001).

7. Tavares, J. et al. Role of host cell traversal by the malaria sporozoite during liver infection. J. Exp. Med. 210, 905-915 (2013).

8. Prudêncio, M., Rodriguez, A. \& Mota, M. M. The silent path to thousands of merozoites: the Plasmodium liver stage. Nat. Rev. Microbiol. 4, 849-856 (2006).

9. Cowman, A. F., Berry, D. \& Baum, J. The cellular and molecular basis for malaria parasite invasion of the human red blood cell. J. Cell Biol. 198, 961-971 (2012).

10. Arredondo, S. A., Schepis, A., Reynolds, L. \& Kappe, S. H. I. Secretory organelle function in the plasmodium sporozoite. Trends Parasitol. https://doi.org/10.1016/j.pt.2021.01.008 (2021).

11. Sultan, A. A. et al. TRAP is necessary for gliding motility and infectivity of plasmodium sporozoites. Cell 90, 511-522 (1997).

12. Buscaglia, C. A., Coppens, I., Hol, W. G. J. \& Nussenzweig, V. Sites of interaction between aldolase and thrombospondin-related anonymous protein in Plasmodium. Mol. Biol. Cell 14, 4947-4957 (2003).

13. Bergman, L. W. et al. Myosin A tail domain interacting protein (MTIP) localizes to the inner membrane complex of Plasmodium sporozoites. J. Cell Sci. 116, 39-49 (2003).

14. Ejigiri, I. et al. Shedding of TRAP by a Rhomboid Protease from the Malaria Sporozoite Surface Is Essential for Gliding Motility and Sporozoite Infectivity. PLoS Pathog. 8, (2012).

15. Gantt, S. et al. Antibodies against thrombospondin-related anonymous protein do not inhibit Plasmodium sporozoite infectivity in vivo. Infect. Immun. 68, 3667-3673 (2000).

16. Matuschewski, K., Nunes, A. C., Nussenzweig, V. \& Menard, R. Plasmodium sporozoite invasion into insect and mammalian cells is directed by the same dual binding system. EMBO J. 21, 1597-1606 (2002).

17. Klug, D. et al. Evolutionarily distant I domains can functionally replace the essential ligand-binding domain of Plasmodium TRAP. Elife 9 (2020).

18. Jethwaney, D. et al. Fetuin-A, a hepatocyte-specific protein that binds Plasmodium berghei thrombospondin-related adhesive protein: a potential role in infectivity. Infect. Immun. 73, 5883-5891 (2005).

19. Dundas, K. et al. Alpha-v-containing integrins are host receptors for the Plasmodium falciparum sporozoite surface protein, TRAP. Proc. Natl. Acad. Sci. https://doi.org/10.1073/pnas.1719660115 (2018).

20. Morahan, B. J., Wang, L. \& Coppel, R. L. No TRAP, no invasion. Trends Parasitol. 25, 77-84 (2009).

21. Song, G. J., Koksal, A. C., Lu, C. F. \& Springer, T. A. Shape change in the receptor for gliding motility in Plasmodium sporozoites. Proc. Natl. Acad. Sci. U. S. A. 109, 21420-21425 (2012).

22. Hodgson, S. H. et al. Evaluation of the efficacy of ChAd63-MVA vectored vaccines expressing circumsporozoite protein and METRAP against controlled human malaria infection in Malaria-Naive individuals. J. Infect. Dis. 211, 1076-1086 (2015).

23. Ogwang, C. et al. Prime-boost vaccination with chimpanzee adenovirus and modified vaccinia Ankara encoding TRAP provides partial protection against Plasmodium falciparum infection in Kenyan adults. Sci. Transl. Med. 7 (2015).

24. Cabral-Miranda, G. et al. Virus-Like Particle (VLP) Plus microcrystalline tyrosine (MCT) adjuvants enhance vaccine efficacy improving T and B cell immunogenicity and protection against Plasmodium berghei/vivax. Vaccines 5 (2017).

25. Kester, K. E. et al. Sequential Phase 1 and Phase 2 randomized, controlled trials of the safety, immunogenicity and efficacy of combined pre-erythrocytic vaccine antigens RTS, S and TRAP formulated with AS02 adjuvant system in healthy, malaria naïve adults. Vaccine 32, 6683-6691 (2014).

26. Bauza, K. et al. Efficacy of a Plasmodium vivax malaria vaccine using ChAd63 and modified vaccinia Ankara expressing thrombospondin-related anonymous protein as assessed with transgenic Plasmodium berghei parasites. Infect. Immun. 82, 1277-1286 (2014).

27. Shen, J. et al. Development of a fully human anti-PDGFRbeta antibody that suppresses growth of human tumor xenografts and enhances antitumor activity of an anti-VEGFR2 antibody. Neoplasia 11, 594-604 (2009).

28. Vaughan, A. M. \& Kappe, S. H. I. Malaria Parasite Liver Infection and Exoerythrocytic Biology. Cold Spring Harb. Perspect. Med. 7, (2017).

29. Silvie, O. et al. Hepatocyte CD81 is required for Plasmodium falciparum and Plasmodium yoelii sporozoite infectivity. Nat. Med. 9, 93-96 (2003).

30. Rodrigues, C. D. et al. Host scavenger receptor SR-BI plays a dual role in the establishment of malaria parasite liver infection. Cell Host Microbe 4, 271-282 (2008).

31. Kaushansky, A. et al. Malaria parasites target the hepatocyte receptor EphA2 for successful host infection. Science 350, 1089-1092 (2015).

32. Langlois, A.-C., Marinach, C., Manzoni, G. \& Silvie, O. Plasmodium sporozoites can invade hepatocytic cells independently of the Ephrin receptor A2. PLoS ONE 13, e0200032 (2018).

33. Miyazaki, H. et al. Expression of platelet-derived growth factor receptor $\beta$ is maintained by Proxl in lymphatic endothelial cells and is required for tumor lymphangiogenesis. Cancer Sci. 105, 1116-1123 (2014).

34. Andrae, J., Gallini, R. \& Betsholtz, C. Role of platelet-derived growth factors in physiology and medicine. Genes Dev. 22, 1276-1312 (2008).

35. Kazlauskas, A. PDGFs and their receptors. Gene 614, 1-7 (2017).

36. Werner, S. \& Grose, R. Regulation of wound healing by growth factors and cytokines. Physiol. Rev. 83, 835-870 (2003).

37. Manzoni, G. et al. Plasmodium P36 determines host cell receptor usage during sporozoite invasion. Elife 6 (2017).

38. Harupa, A. et al. SSP3 is a novel plasmodium yoelii sporozoite surface protein with a role in gliding motility. Infect. Immun. 82, 4643-4653 (2014)

39. Carbonetti, S. et al. A method for the isolation and characterization of functional murine monoclonal antibodies by single B cell cloning. J. Immunol. Methods 448, 66-73 (2017).

40. Wang, J.-Y. et al. Improved expression of secretory and trimeric proteins in mammalian cells via the introduction of a new trimer motif and a mutant of the tPA signal sequence. Appl. Microbiol. Biotechnol. 91, 731-740 (2011).

41. Beckett, D., Kovaleva, E. \& Schatz, P. J. A minimal peptide substrate in biotin holoenzyme synthetase-catalyzed biotinylation. Protein Sci. 8, 921-929 (1999).

42. Swearingen, K. E. et al. Proteogenomic analysis of the total and surface-exposed proteomes of Plasmodium vivax salivary gland sporozoites. PLoS Negl. Trop. Dis. 11, e0005791 (2017).

43. Carlton, J. M. et al. Comparative genomics of the neglected human malaria parasite Plasmodium vivax. Nature 455, 757-763 (2008).

44. Auburn, S. et al. A new Plasmodium vivax reference sequence with improved assembly of the subtelomeres reveals an abundance of pir genes. Wellcome Open Res 1, 4 (2016).

45. Jex, A. et al. Integrated transcriptomic, proteomic and epigenomic analysis of Plasmodium vivax salivary-gland sporozoites. BioRxiv https://doi.org/10.1101/145250 (2017).

46. von Itzstein, M., Plebanski, M., Cooke, B. M. \& Coppel, R. L. Hot, sweet and sticky: the glycobiology of Plasmodium falciparum. Trends Parasitol. 24, 210-218 (2008). 
47. Bushkin, G. G. et al. Suggestive evidence for Darwinian Selection against asparagine-linked glycans of Plasmodium falciparum and Toxoplasma gondii. Eukaryot. Cell 9, 228-241 (2010).

48. Carbonetti, S., Oliver, B. G., Glenn, J., Stamatatos, L. \& Sather, D. N. Soluble HIV-1 envelope immunogens derived from an elite neutralizer elicit cross-reactive V1V2 antibodies and low potency neutralizing antibodies. PLoS ONE 9, e86905 (2014).

49. Backliwal, G., Hildinger, M., Hasija, V. \& Wurm, F. M. High-density transfection with HEK-293 cells allows doubling of transient titers and removes need for a priori DNA complex formation with PEI. Biotechnol. Bioeng. 99, 721-727 (2008).

50. Miller, J. L. et al. Quantitative bioluminescent imaging of pre-erythrocytic malaria parasite infection using luciferase-expressing Plasmodium yoelii. PLoS ONE 8, e60820 (2013).

51. Foquet, L. et al. Anti-CD81 but not anti-SR-BI blocks Plasmodium falciparum liver infection in a humanized mouse model. J. Antimicrob. Chemother. 70, 1784-1787 (2015).

52. Sack, B. K. et al. Model for in vivo assessment of humoral protection against malaria sporozoite challenge by passive transfer of monoclonal antibodies and immune serum. Infect. Immun. 82, 808-817 (2014).

\section{Acknowledgements}

We would like to thank the Seattle Children's Research Institute insectary staff, principally Heather Kain, for the help with mosquito production. We also thank Drs. S. Mikolajczak and E. Flannery (Novartis Institute for Tropical Diseases) for assistance with the design of the P. vivax TRAP protein.

\section{Author contributions}

R.W.J.S., V.V., D.N.S. and S.H.I.K. designed the study. K.E.S. and R.L.M. designed Plasmodium vivax source sequences. V.V., R.W.J.S. and S.C. generated protein-encoding constructs. V.V. and N.D. produced recombinant proteins and performed protein binding studies. J.S., H.T. and J.F. designed and performed the cell array protein binding screen. R.W.J.S. and V.V. performed flow-cytometry binding studies. R.W.J.S., S.A.A., D.G., S.K. and B.S.F. performed the immunohistochemistry experiments. R.W.J.S., B.K.W., T.N., W.B. and N.C., performed the in vivo studies. R.W.J.S. and V.V. wrote the paper. D.N.S. and S.H.I.K. supervised the project, secured the funding and edited the manuscript.

\section{Funding}

Our work was supported by National Institutes of Health Grants R01AI134956 (S.H.I.K), R01AI117234 (D.N.S. and S.H.I.K), K25AI119229 (K.E.S), R01GM087221 (R.L.M.). The content is solely the responsibility of the authors and does not necessarily represent the official views of the National Institutes of Health.

\section{Competing interests}

The authors declare no competing interests.

\section{Additional information}

Supplementary Information The online version contains supplementary material available at https://doi.org/ 10.1038/s41598-021-90722-5.

Correspondence and requests for materials should be addressed to D.N.S. or S.H.I.K.

Reprints and permissions information is available at www.nature.com/reprints.

Publisher's note Springer Nature remains neutral with regard to jurisdictional claims in published maps and institutional affiliations.

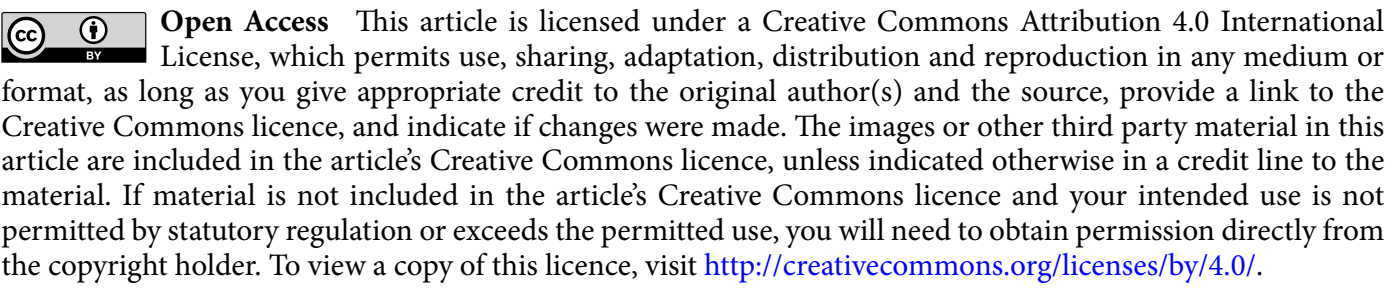

(c) The Author(s) 2021 